\title{
Checklist of the Sipunculan Species
}

Class Sipunculidea

Order Sipunculiformes

Family Sipunculidae

Sipunculus

Sipunculus (Sipunculus) lomonossovi, longipapillosus, marcusi, norvegicus, nudus, phalloides phalloides, p. inclusus, polymyotus, robustus

Sipunculus (Austrosiphon) indicus, mundanus

Xenosiphon absconditus, branchiatus

Siphonosoma arcassonense, australe australe, a. takatsukii, boholense, cumanense, dayi, funafuti, ingens, mourense, ro-

tumanum, vastum

Siphonomecus multicinctus

Phascolopsis gouldii

Order Golfingiiformes

Family Golfingiidae

Golfingia

Golfingia (Spinata) pectinatoides

Golfingia (Golfingia) anderssoni, birsteini, capensis, elongata, iniqua, margaritacea margaritacea, m. ohlini, mirabilis, muricaudata, vulgaris vulgaris, v. herdmani

Nephasoma

Nephasoma (Cutlerensis) rutilofuscum

Nephasoma (Nephasoma) abyssorum abyssorum, a benhami, bulbosum, capilleforme, confusum, constricticervix, constrictum, cutleri, diaphanes diaphanes, $d$. corrugatum, eremita, filiforme, flagriferum, laetmophilum, lilljeborgi, minutum, multiaraneusa, novaezealandiae, pellucidum pellucidum, $p$. subhamatum, rimicola, schuettei, tasmaniense, vitjazi, wodjanizkii wodjanizkii, w. elisae

Thysanocardia catharinae, nigra, procera 
Family Phascolionidae

Phascolion

Phascolion (Isomya) convestitum, gerardi, hedraeum, lucifugax, microspheroidis, tuberculosum

Phascolion (Lesenka) collare, cryptum, hupferi, rectum, valdiviae valdiviae, $v$. sumatrense

Phascolion (Montuga) lutense, pacificum

Phascolion (Phascolion) abnorme, bogorovi, caupo, hibridum, medusae, megaethi, pharetratum, psammophilus, robertsoni, strombus strombus, s. cronullae, ushakovi

Phascolion (Villiophora) cirratum

Onchnesoma intermedium, magnibathum, squamatum squamatum, s. oligopapillosum, steenstrupii steenstrupii, $s$. nudum

Family Themistidae

Themiste

Themiste (Themiste) alutacea, blanda, dyscrita, hennahi, pyroides

Themiste (Lagenopsis) cymodoceae, dehamata, lageniformis, minor minor, $m$. huttoni, variospinosa

Class Phascolosomatidea

Order Phascolosomatiformes

Family Phascolosomatidae

Phascolosoma

Phascolosoma (Fisherana) capitatum, lobostomum

Phascolosoma (Phascolosoma) agassizii agassizii, a. kurilense, albolineatum, annulatum, arcuatum, glabrum glabrum, g. multiannulatum, granulatum, maculatum, meteori, nigrescens, noduliferum, pacificum, perlucens, saprophagicum, scolops, stephensoni, turnerae

Antillesoma antillarum

Apionsoma

Apionsoma (Apionsoma) misakianum, murinae murinae, m. bilobatae, trichocephalus

Apionsoma (Edmondsius) pectinatum

Order Aspidosiphoniformes

Family Aspidosiphonidae

Aspidosiphon

Aspidosiphon (Akrikos) albus, mexicanus, thomassini, venabulum, zinni 
Aspidosiphon (Aspidosiphon) elegans, exiguus, gosnoldi, gracilis gracilis, g. schnehageni, misakiensis, muelleri, spiralis Aspidosiphon (Paraspidosiphon) coyi, fischeri, laevis, parvulus, planoscutatus, steenstrupii, tenuis

Lithacrosiphon cristatus, cristatus lakshadweepensis, maldivensis Cloeosiphon aspergillus 

The Sipuncula 
